Comparación del comienzo del efecto de los antidepresivos: consideraciones pragmáticas sobre los métodos y los puntos finales.

D. Hackett

Comparación del comienzo de la acción de los antidepresivos: aplicación de diferentes criterios al mismo conjunto de datos.

I. A. Niklson, P. E. Reimitz .

Comienzo de la mejoría con fluoxetina y moclobemida.

H. H. Stassen, J. Angst, A. Delini-Stula.

Un enfoque de análisis de regresión para estimar el comienzo de la actividad de los antidepresivos.

H. Müller, H. J. Möller ....

Sensibilidad de las escalas de evaluación del observador por el análisis del número de días hasta la mejoría en pacientes con depresión mayor.

N. Lauge, K. Behnke, J. Sogaard, B. Bahr, P. Bech.

Calidad de vida en la esquizofrenia: seguimiento a largo plazo en 362 pacientes esquizofrénicos crónicos ambulatorios españoles que recibian tratamiento de mantenimiento de risperidona.

J. Bobes, M. Gutiérrez, J. Gibert, M. P. González, L. Herraiz, A. Fernández ………....................... Algunas consideraciones estadísticas en el desarrollo de nuevas escalas de calidad de vida (QoL) en la esquizofrenia.

J. McKellar.

Aspectos particulares de la evaluación de acontecimientos adversos en la vigilancia postcomercialización.

D. Baier. 

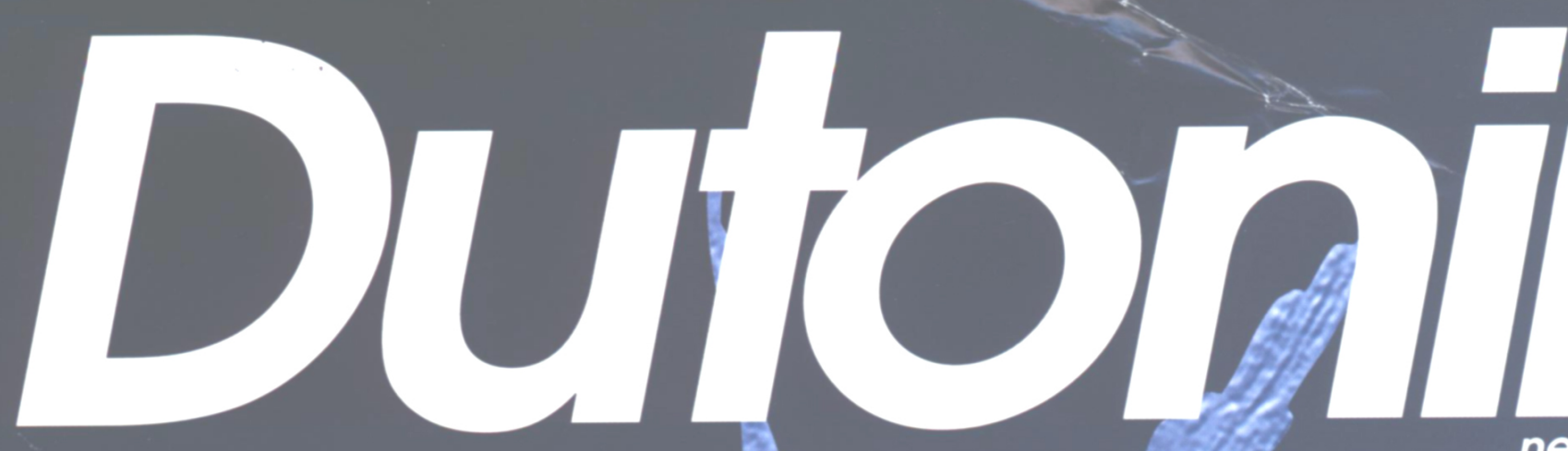

\section{Eficacia y calidad de vidó en el tratamiento de la depresión}

Eficacia antidepresiva comparable a la de los ATC o ISRS '

Mejora la calidad del sueño sin producir sedación ${ }^{2,3}$

Reduce los sintomas de ansiedad asociados a la depresión ${ }^{4}$

\begin{abstract}
Presenta menor incidencia de sintomas de activación (ansiedad, agitación e insomnio) que los ISRS y venlafaxina ${ }^{5}$
\end{abstract}

No modifica el peso del paciente

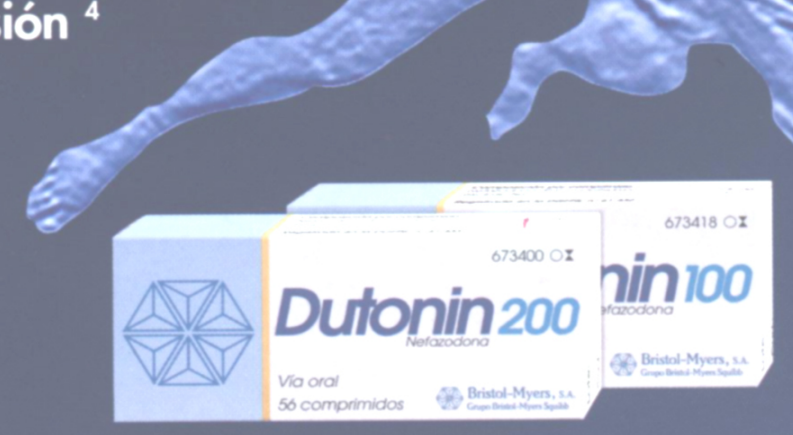

\section{Preserva la función sexual ${ }^{7}$}

DUTONINQ. (Nefarodona). $50 \mathrm{mg}, 100 \mathrm{mg}$ y $200 \mathrm{mg}$

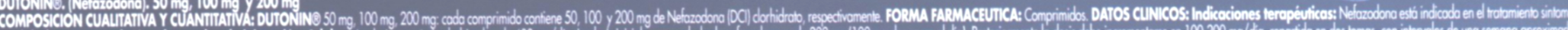

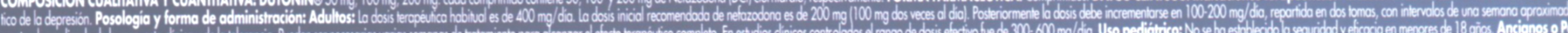

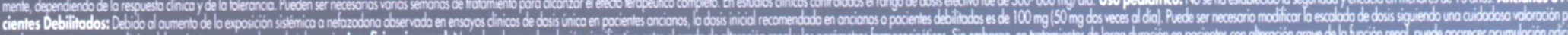

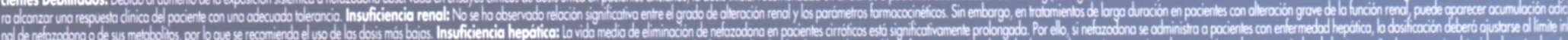

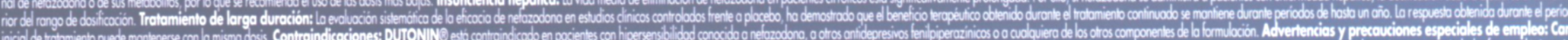

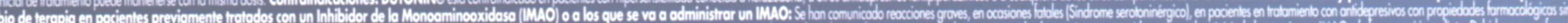

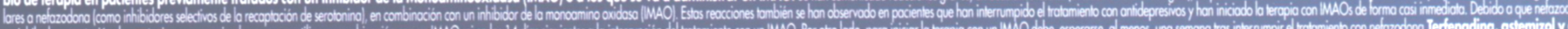

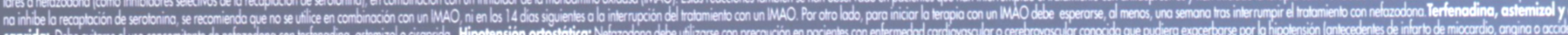

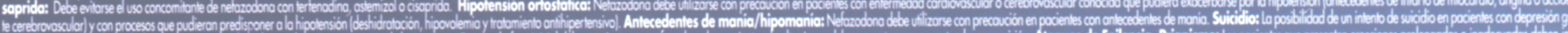

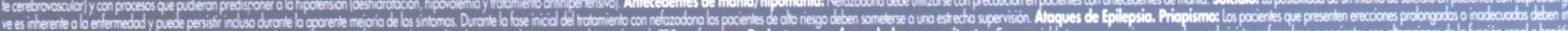

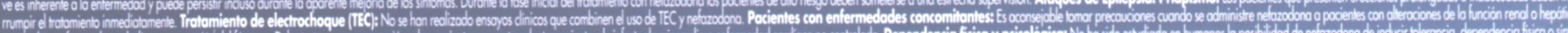

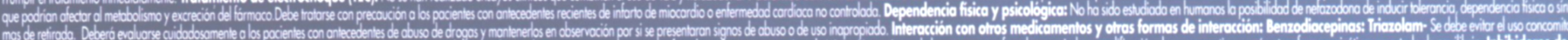

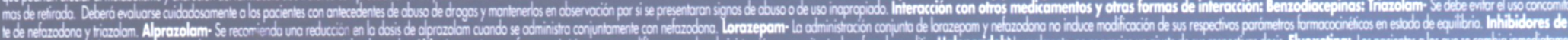

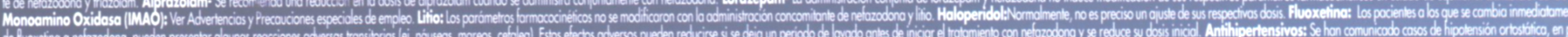

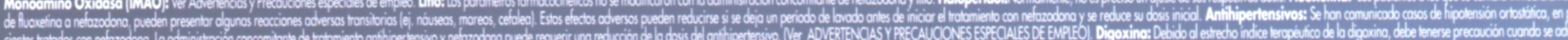

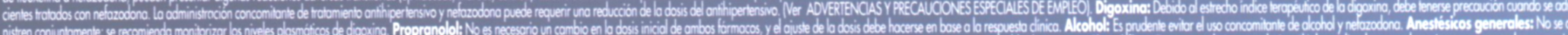

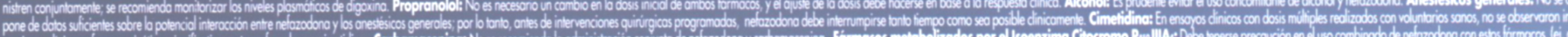

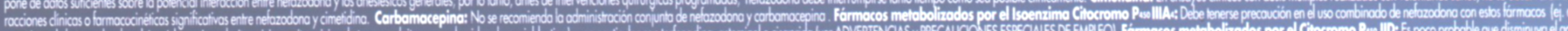

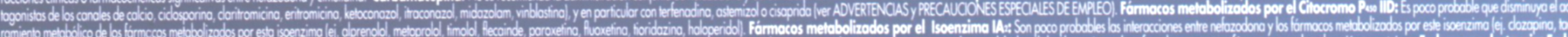

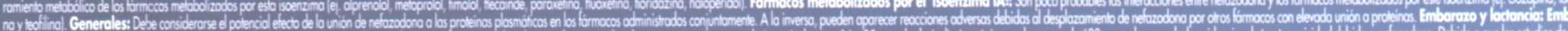

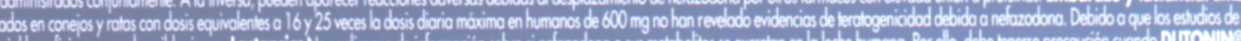

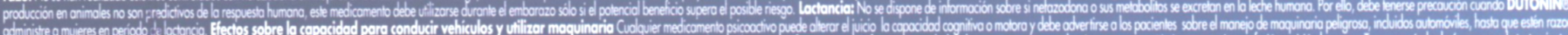

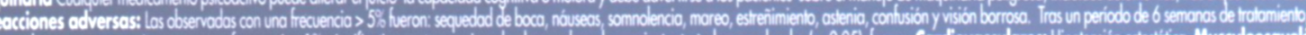

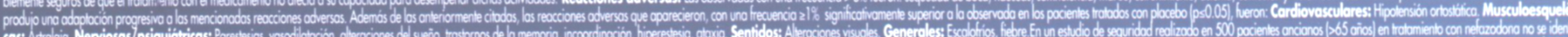

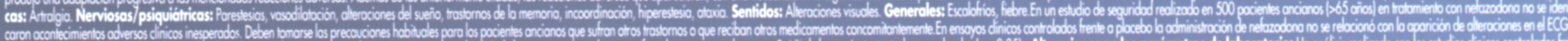

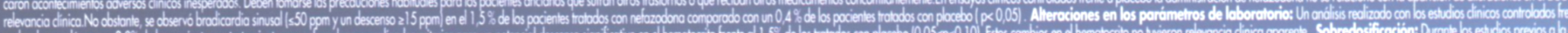

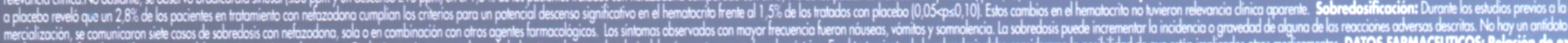

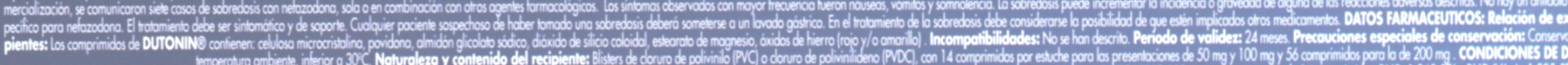

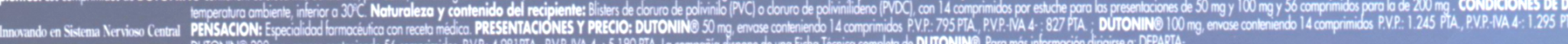

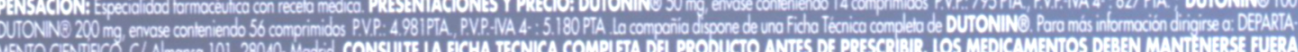

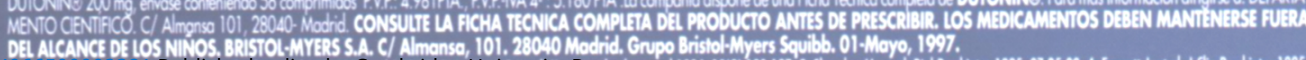

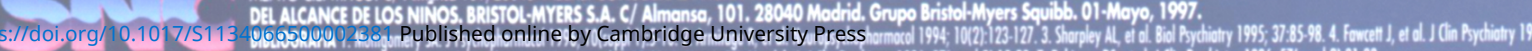

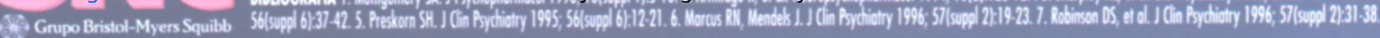

Grupo Bristol-Myers Squib Comprometidos con la salud 


\section{EUROPEAN PSYCHIATRY}

Editors-in-chief: P Boyer, Y. Lecrubier (Paris).

Secretariat: Hôpital de la Salpêtrière, INSERM U 302, Pavillon Clérambault, 47, bd de l'Hôpital, 75651 Paris cedex 13, France.

Tel: (33) 0142161656 . Fax: (33) 0145852800

Editors: JD Guelfi (Paris/Villejuif), R. Murray (London), M Maj (Naples), CB Pull (Luxembourg), M. Ackenheil (Munich), P Bech (Copenhagen)

Editors Emeritus: C Ballus (Barcelona), H Heimann (Tübingen)

\section{Consejo Editorial}

J Adès, Colombes, France

HS Akiskal, Rockville, MD, USA

NC Andreasen, lowa City, IA, USA

J Angst, Zurich, Switzerland

M. Ansseau, Liège, Belgium

P Baumann, Lausanne, Switzerland

H Beckmann, Würzburg, Germany

P Berner, Vienna, Austria

A Bertelsen, Risskov, Denmark

J Biber, Cadiz, Spain

J Birley, London, UK

JC Bisserbe, Meudon, France

B Bondy, Munich, Germany

JP Boulenger, Sherbrooke, Canada

M Bourgeois, Bordeaux, France

F Brambilla, Milan, Italy

I Brockington, Birmingham, UK

A Clare, Dublin, Ireland

F Clerget-Darpoux, Paris, France

V Conde Lopez, Valladolid, Spain

S Consoli, Paris, France

P Cosyns, Antwerp, Belgium

J Cottraux, Lyon France

$M$ von Cranach, Kaufbeuren, Germany

A Dahl, Oslo, Norway

JM Danion, Strasbourg, France

JFW Deakin, Manchester, UK

$\mathrm{M}$ de Bonis, Le Kremlin Bicêtre, Paris, France

$\mathrm{H}$ Dufour, Lausanne, Switzerland

R Engel, Munich, Germany

T Fahy, Galway, Ireland

L Farde, Stockholm, Sweden

A Féline, Le Kremlin Bicêtre, Paris, France

A Fernandes da Fonseca, Porto, Portugal

F Ferrero, Geneva, Switzerland
M Fichter, Prien am Chiemsee, Germany

H Freeman, London, $U K$

HJ Gaertner, Tübingen, Germany

D Goldberg, Manchester, UK

I Hand, Hamburg, Germany

H Häfner, Mannheim, Germany

T Helgason, Reykjavik, Iceland

H Hippius, Munich, Germany

A Jablenski, Sofia, Bulgaria

E Johnston, Edinburgh, $U K$

S Kasper, Vienna, Austria

M Kastrup, Hvidovre, Denmark

D Kemali, Naples, Italy

R Kendell, Edinburgh, $U K$

D Klein, New York, NY, USA

R Klein, New York, NY, USA

$S$ Langer, Paris, France

J Lellouch, Villejuif, France

P Lemoine, Lyon, France

T Lemperière, Colombes, France

JP Lépine, Paris, France

OM Lesch, Vienna, Austria

SW Lewis, London, UK

H Lôo, París, France

JJ López-Ibor, Madrid, Spain

P McGuffin, Cardiff, $U K$

W Maier, Mainz, Germany

A Mann, London, UK

K Mann, Tübingen, Germany

I Marks, London, UK

J Marlet, Venray, The Netherlands

J Massanna, Barcelona, Spain

J Mendlewicz, Brussels, Belgium

HJ Möller, Munich, Germany

N Müller, Munich, Germany
M Musalek, Vienna, Austria

D Naber, Munich, Germany

E O'Callaghan, Dublin, Ireland

Y Ono, Tokyo, Japan

M Patris, Strasbourg, France

J Pellet, Saint-Etienne, France

C Perris, Umeä, Sweden

P Pichot, Paris, France

T Pohlmächer, Munich, Germany

$\mathrm{H}$ Pope, Belmont, MA, USA

AJ Puech, Paris, France

G Racagni, Milan, Italy

N Retterstøl, Oslo, Norway

M A Ron, London, UK

R Rosenberg, Risskov, Denmark

M Roth, Cambridge, UK

F Rouillon, Colombes, France

J Saiz-Ruiz, Madrid, Spain

A Sánchez-Blanque, Zaragoza, Spain

N Sartorius, Geneva, Switzerland

F Schulsinger, Copenhagen, Denmark

G Sedvall, Stockholm, Sweden

L Singer, Strasbourg, France

CN Stefanis, Athens, Greece

E Straube, Tübingen, Germany

E Taylor, London, $U K$

$\mathrm{P}$ Taylor, London, $U K$

L Träskman-Bendz, Lund, Sweden

J Vallejo, Barcelona, Spain

L Waintraub, Paris, France

D Widlöcher, Paris, France

J Wilmotte, Charleroi, Belgium

J Wing, London, $U K$

FT Zimmer, Tübingen, Germany

J Zohar, Beer-Sheva, Israel

\section{Association of European Psychiatrists}

President: J Angst (Zürich); Past President: R Murray (London); President Elect: N Sartorius (Geneva); Secretary General: CB Pull (Luxembourg); Treasurer: M Patris (Strasbourg); Counsellors: H Häfner (Mannheim), Y Lecrubier (Paris); Section: L Singer (Strasbourg).

European Psychiatry, edición original, es publicada por Editions Scientifiques Elsevier, 141, rue de Javel, 75747 París, Francia. Indexada en: Biological Abstracts/Biosis, CNRS/Pscal, Current Contents/Clinical Medicine and Social Behavioral Sciences, Excerpta Medica/EMbase, Psychological Abstracts

\section{Director de la Edición Española: C. Ballús}

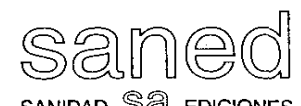

Sanidad y Ediciones (SANED, S.A.). C/ Paseo de la Habana, 202 bis. 28036 MADRID. Telf.: (91) 3594092.

Caspe, 172, 4. ${ }^{\circ}$-A. BARCELONA. Telf.: (93) 2472411.

Directora Editorial: Alicia Martínez Magro.

Suscripciones: SANED, S.A. Paseo de la Habana, 202 bis. 28036 MADRID.

Publicación Bimensual ( 8 números al año).

Composición: Artecomp, S.L. Albarracín, 50-1.․ Madrid. Imprime: D.g.B. Resina, 13-15. Madrid.

Soporte válido M. Sanidad: SV 93040 R. ISSN: 1134-0665. Depósito Legal: M-5991-1994.

Copyright de los textos originales 1998. Reservados todos los derechos. Ninguna parte de esta publicación puede ser reproducida, transmitida en ninguna forma o medio alguno; electrónico o mecánico, incluyendo fotocopias, grabaciones o cualquier sistema de recuperación de

almacenaje de información, sin la autorización por escrito de los titulares del Copyright. 

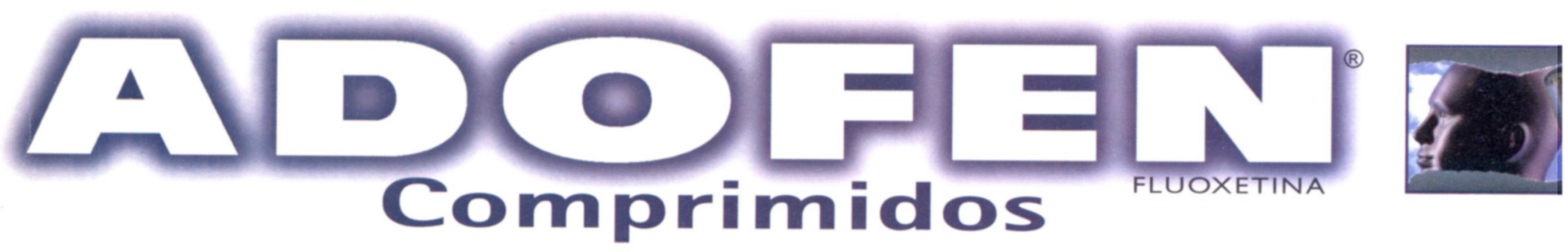

El inhibidor selectivo Patrón en el tratamiento antidepresivo UNICO APROBADO EN LA DEPRESION CON ANSIEDAD ASOCIADA

- DEPRESION

- BULIMIA NERVIOSA

- TRASTORNO OBSESIVO-COMPULSIVO

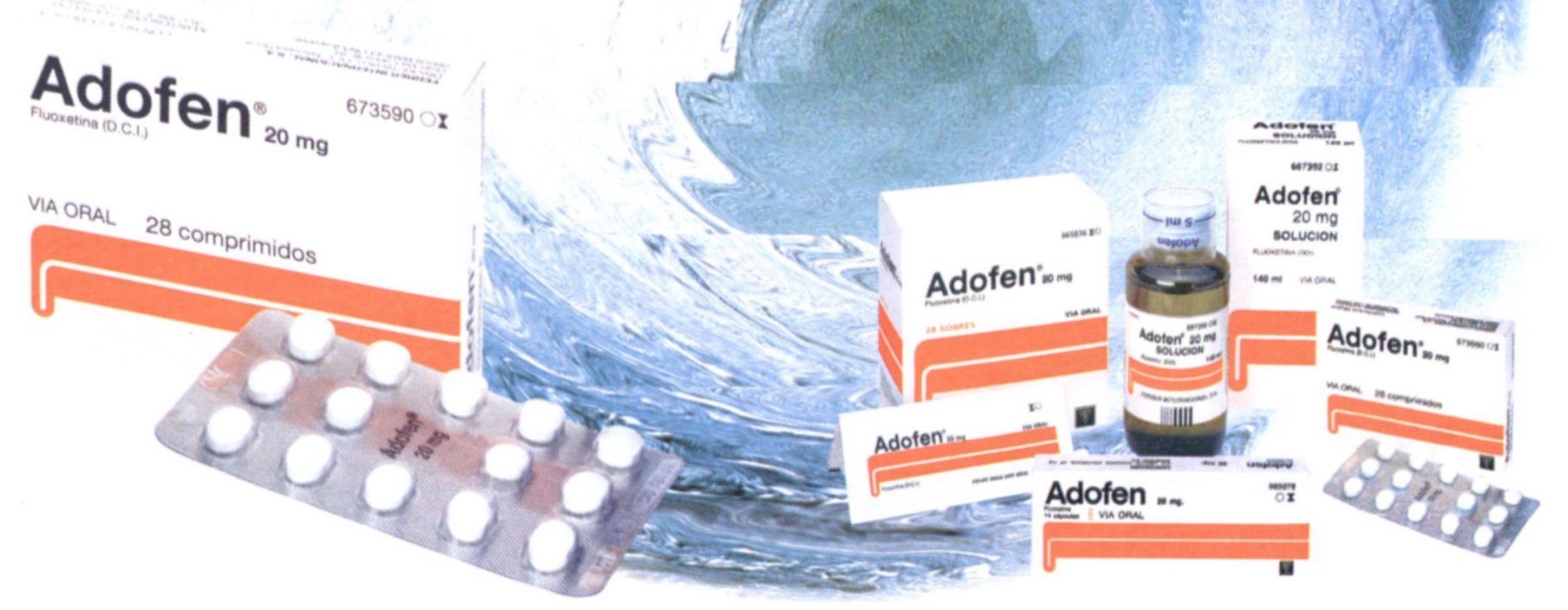

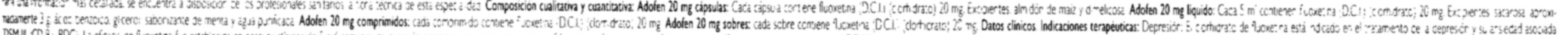

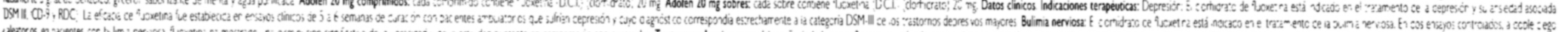

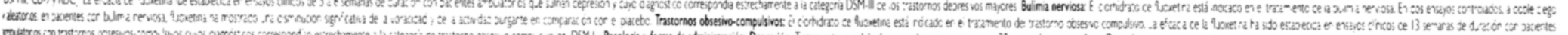

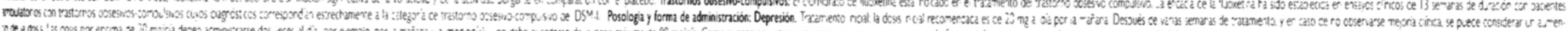

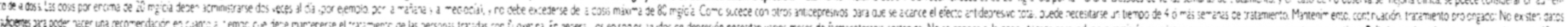

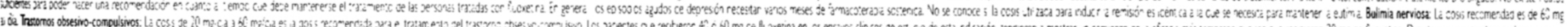

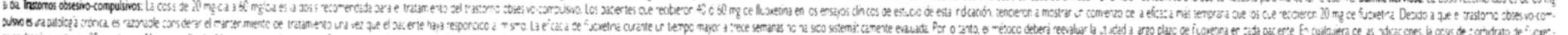

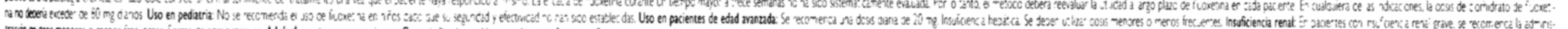

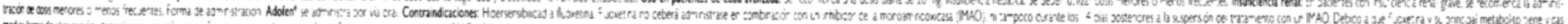

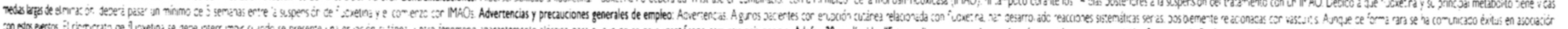

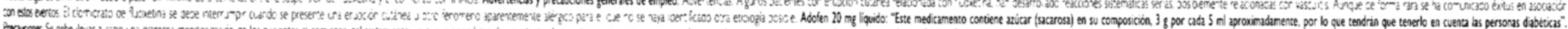

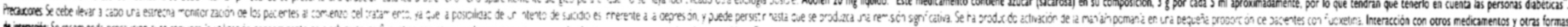

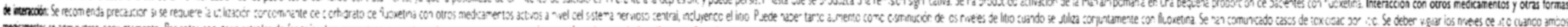

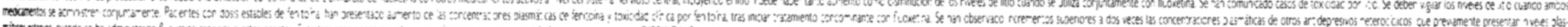

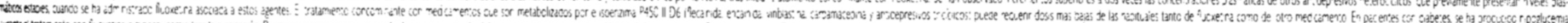

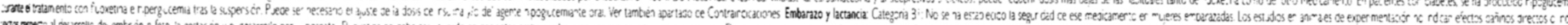

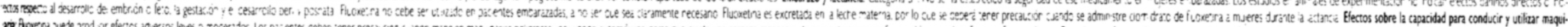

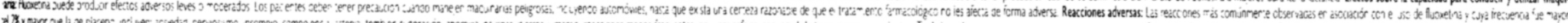

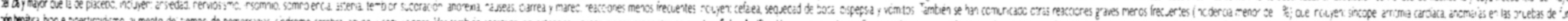

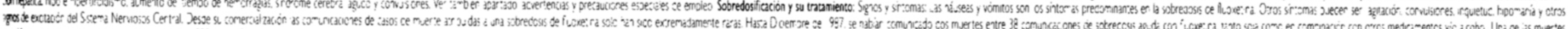

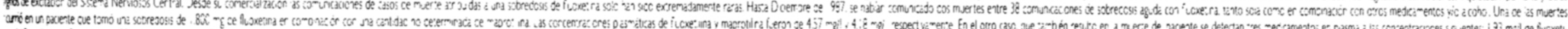

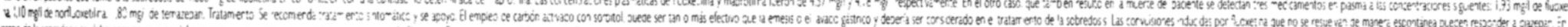

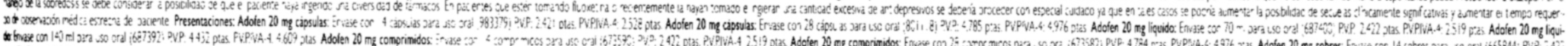

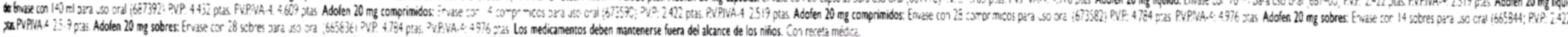




\title{
EUROPEAN PSYCHIATRY.
}

EDICION ESPAÑOLA

\author{
Volumen 5, Número 7, Octubre 1998
}

ECST STRASBOURG, 2-3 JUNIO 1997

Comparación del comienzo del efecto de los antidepresivos: consideraciones pragmáticas sobre los métodos y los puntos finales.

D. Hackett.

Comparación del comienzo de la acción de los antidepresivos: aplicación de diferentes criterios al mismo conjunto de datos.

I. A. Nikison, P. E. Reimitz

Comienzo de la mejoría con fluoxetina y moclobemida.

H. H. Stassen, J. Angst, A. Delini-Stula.

Un enfoque de análisis de regresión para estimar el comienzo de la actividad de los antidepresivos.

H. Müller, H. J. Möller.

Sensibilidad de las escalas de evaluación del observador por el análisis del número de días hasta la mejoría en pacientes con depresión mayor.

N. Lauge, K. Behnke, J. Sogaard, B. Bahr, P. Bech.

Calidad de vida en la esquizofrenia: seguimiento a largo plazo en 362 pacientes esquizofrénicos crónicos ambulatorios españoles que recibian tratamiento de mantenimiento de risperidona.

J. Bobes, M. Gutiérrez, J. Gibert, M. P. González, L. Herraiz, A. Fernández.

Algunas consideraciones estadísticas en el desarrollo de nuevas escalas de calidad de vida (QoL) en la esquizofrenia.

J. McKellar

Aspectos particulares de la evaluación de acontecimientos adversos en la vigilancia postcomercialización.

D. Baier. 


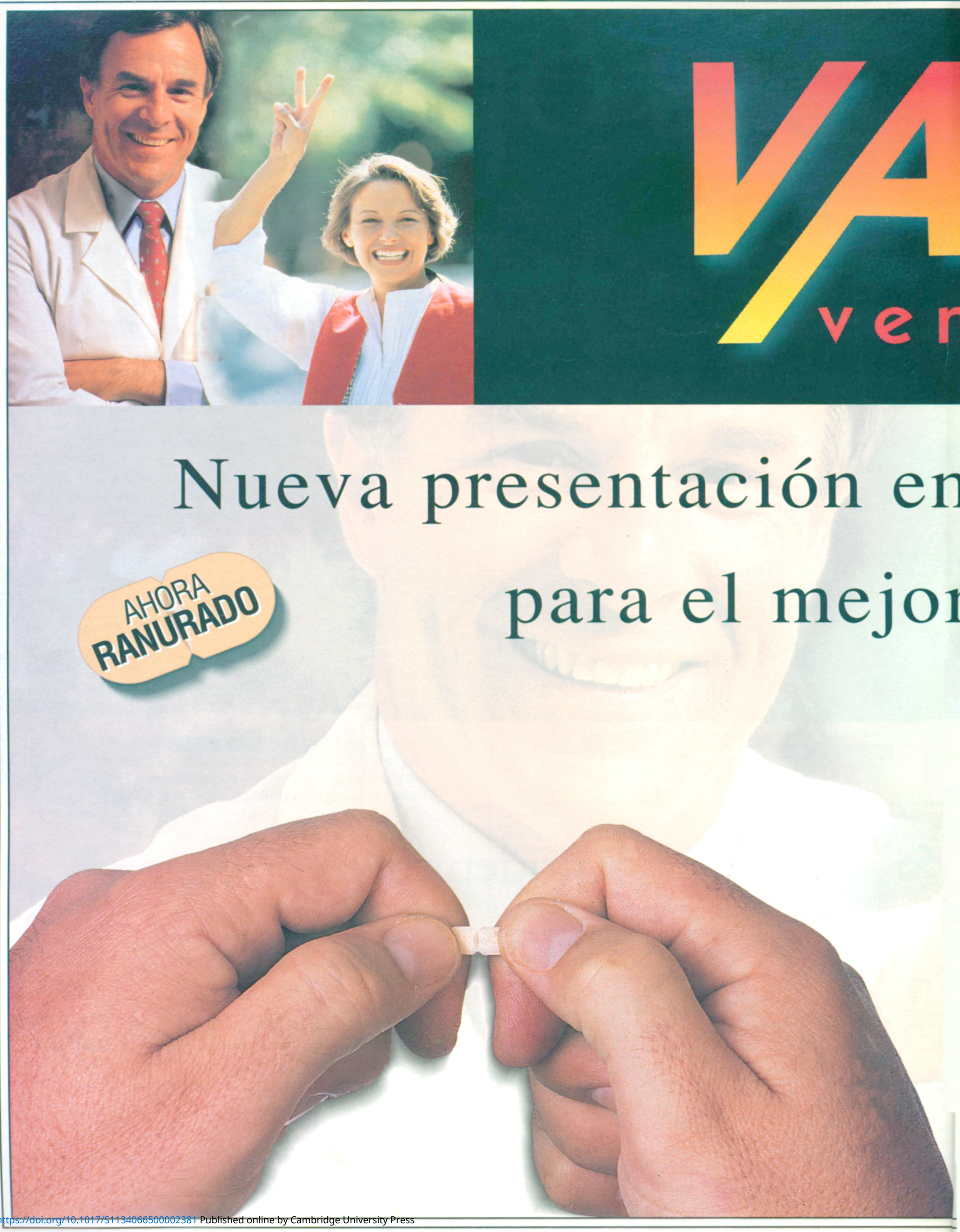





\section{Ahora más que nunca. El antidepresivo de $1^{\text {a }}$ elección.}

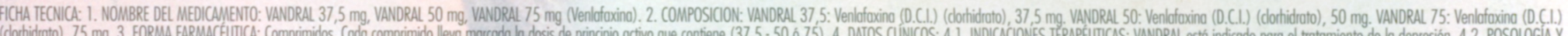

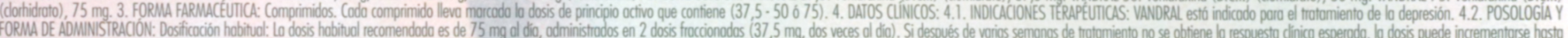

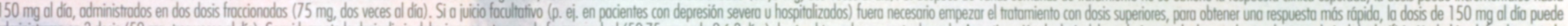

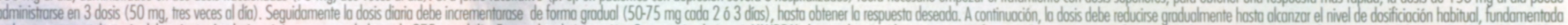

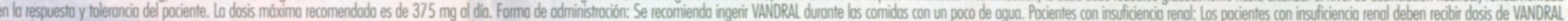

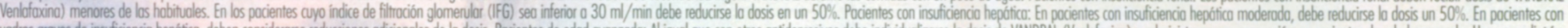

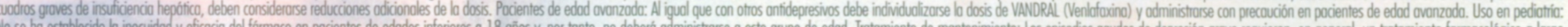

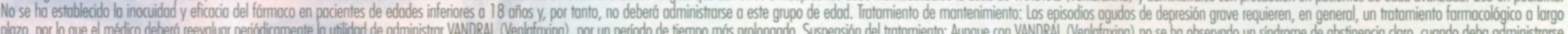

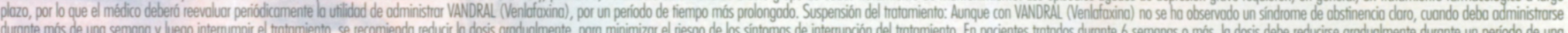

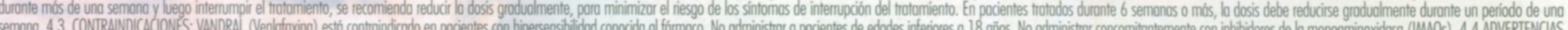

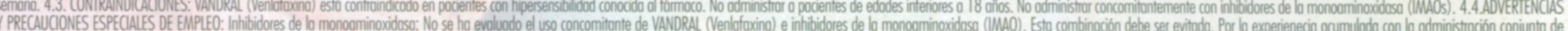

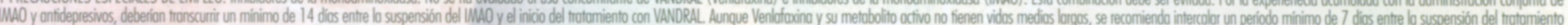

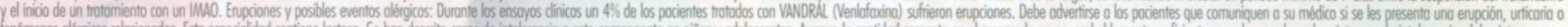

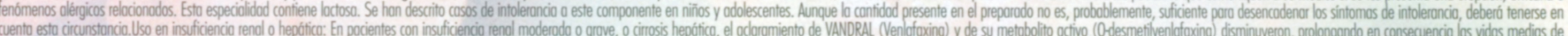

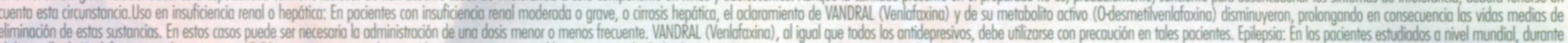

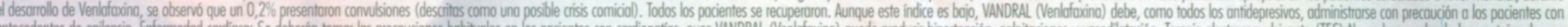

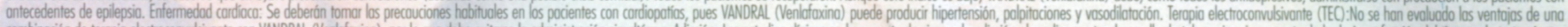

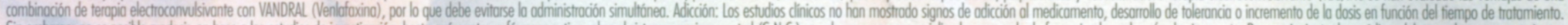

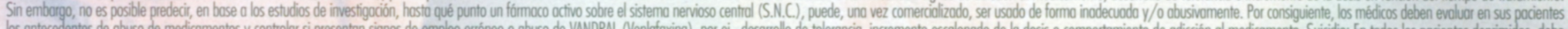

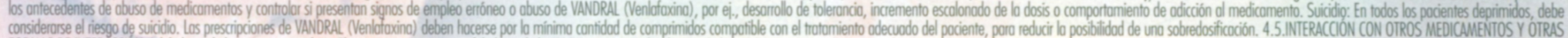

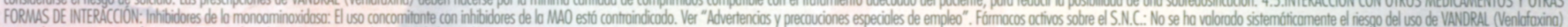

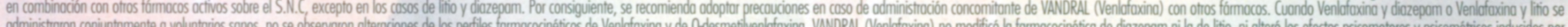

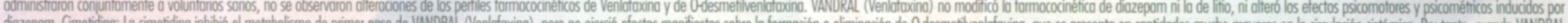

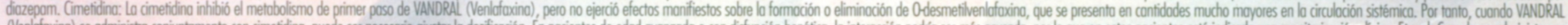

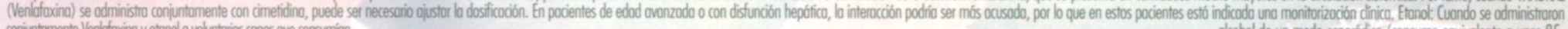
conivitumente Vendfoxino y etond a voluntrios sonos que consumion

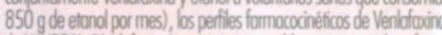

de VANDRAL (Venlafoxina) en régimen estable no potenóól los efectis

bebedores socioles cuando no recibieron VANDRAL (Venlafoxino.

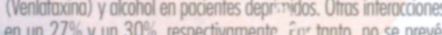

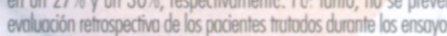

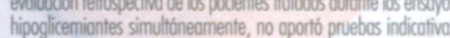

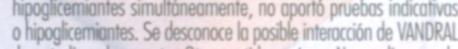
de estudios al respecto. Otros anfidepresivos: No se dispone de de VANDRAL

de emplea". Inhibidores o inductores de los enzimos hepéficos: Ln inhibidor de los enzimas hepóficos, es conveniente reducir lo dosis

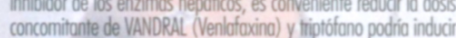

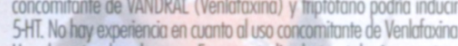
Uso durante el emberazo: En un estudio de teratologio en rotos, (oproximadomente 11 veces lo dosis móxima recomendoda pard fetotoxiódod carocterizado por un retroso del crecimiento, que podio

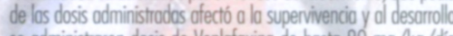
se odministrtoron dosis de Venlofoxino de hosto $90 \mathrm{mg} / \mathrm{kg} / \mathrm{dig}$

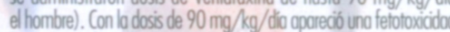

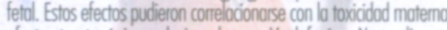
efectos tantogénicos relacionnados con Venloffuino. No se dispore de reproducción onimal no siempre pemiten pronosficar lo respuesto emborazo, cuando los beneficios justifiquen los posit

e encuentron embarozodos o fienen intención de quedorse

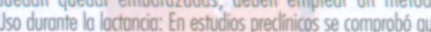

sus metobolitios son excretodos por la leche humano. Por lo tont's

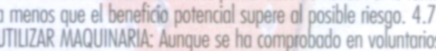

cognoscitivo o el comportumiento compleio, los fómocos psicooctives

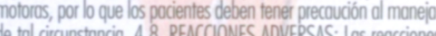

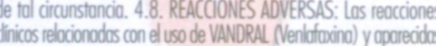

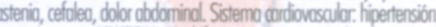

Totariento con VANDRAL (Venlffoxina) se asoób a un ligero oumento

medios de la presión arterial diostólico de aproximndomente 2 man

$\mathrm{Hg}$, aproximodamente) en pocientes thitod
trotodos con plocebo. Se consideró que un 35

inicamente signiticativos de lo presion anteric

de $7 \mathrm{~mm}$ Hg, en la semono 6 . La presesencin

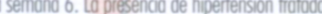

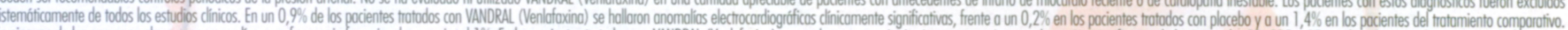

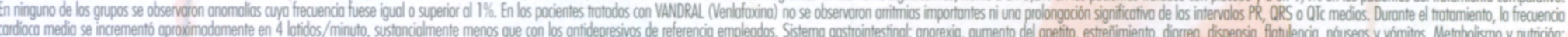

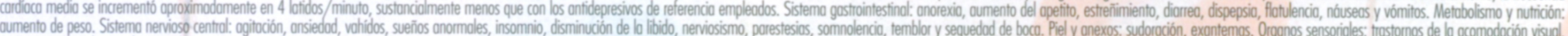

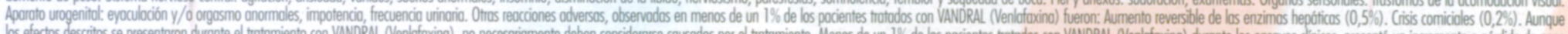

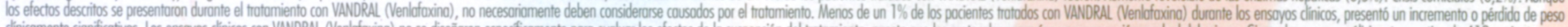

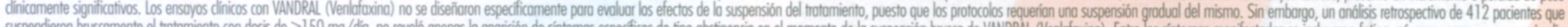

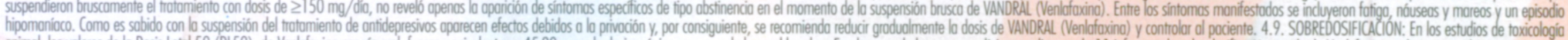

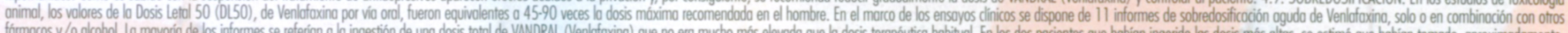

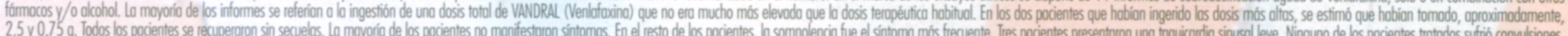

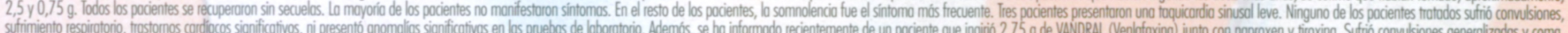

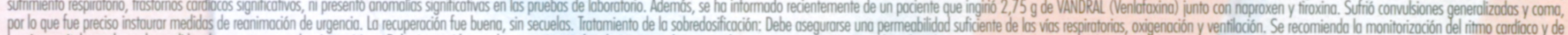

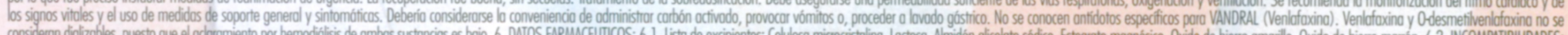

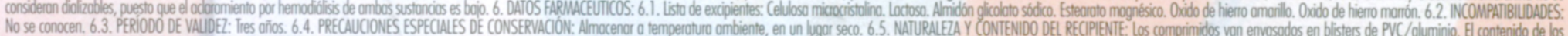

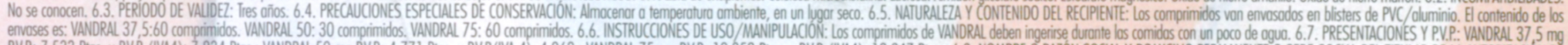

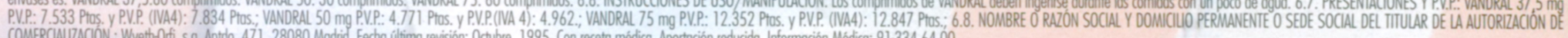

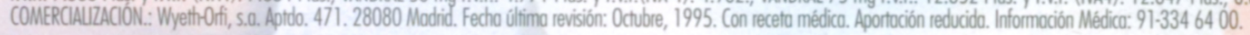



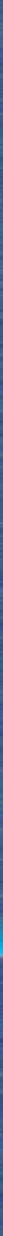

\section{La mayor red nacional de información y comunicaciones sanitarias accesible en Internet}

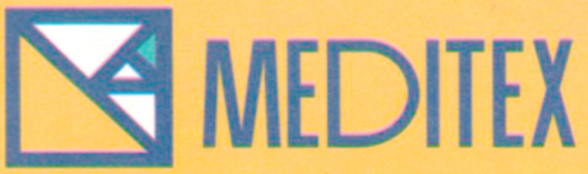

Po de la Castellana, 123 • 28046 MADRID • Telf.: (91) 5558239 • Fax: (91) 5552807 E-Mail: medynet@ medynet.com

C/ Caspe, 172 • 08013 BARCELONA • Telf.: (93) 2472411 • Fax: (93) 2655224 • E-Mail: sanedb @medynet.com https://doi.org/10.1017/S1134066500002381 Published online by Cambridge University Press 

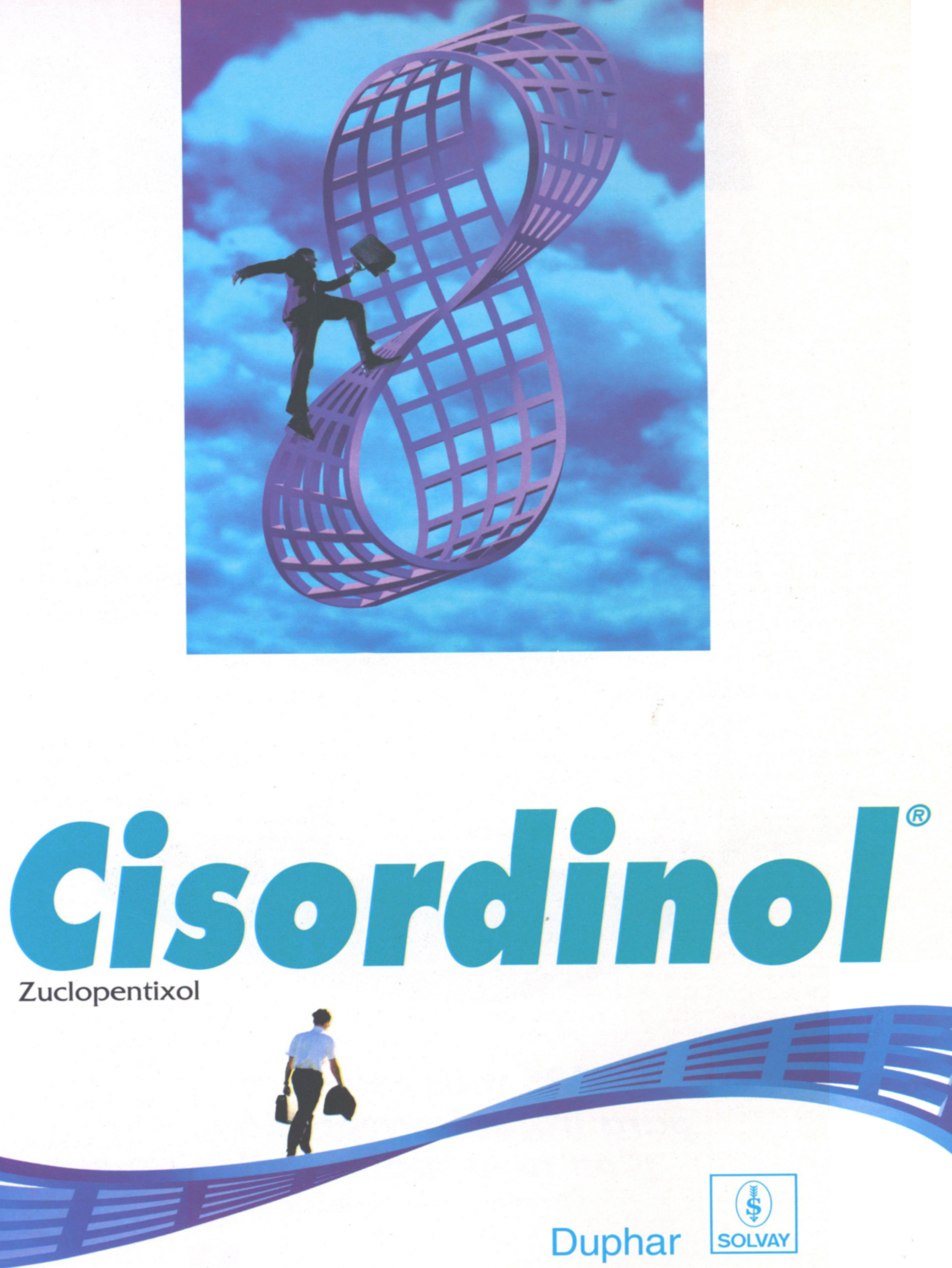


\title{
EUROPEAN PSYCHIATRY.
}

EDICION ESPAÑOLA

\author{
Volume 5, Number 7, October 1998
}

ECST STRASBOURG, 2-3 JUNE 1997

Comparing onset of effect of antidepressants: pragmatic considerations on methods and end-points.

D. Hackett

Comparing the onset of action of antidepressants: comparison of different criteria applied to the same data set.

1. A. Niklson, P. E. Reimitz

Onset of improvement under fluoxetine and moclobemide.

H. H. Stassen, J. Angst, A. Delini-Stula.

A regresion analytical approach to estimate the onset of activity of antidepressants.

H. Müller, H. J. Möller.....

Responsiveness of observer rating scales by analysis of number of days until improvements in patients with major depression.

N. Lauge, K. Behnke, J. Søgaard, B. Bahr, P. Bech..

Quality of life schizophrenia: long-term folow-up in 362 chronic Spanish schizophrenic outpatients undergoing risperidone maintenance treatment.

J. Bobes, M. Gutiérrez, J. Gibert, M. P. González, L. Herraiz, A. Fernández

Some estatistical considerations in the development of new quality of life (QoL) scales in schizophrenia.

J. McKellar.

Particular aspects of adverse event assessment in post-marking surveillance.

D. Baier. 